\title{
Context-Dependence and the Sorites
}

\author{
Graeme Forbes
}

Aвstract: In Section 1 we describe the Sorites paradox and lay out options for a solution. In Section 2 we consider approaches which deny that all premises are true, and note that these solutions all seem open to a certain serious objection. In Section 3 we note a problem for the principle of transitivity of the conditional and present a contextualist resolution of the problem, according to which the "counterexamples" to transitivity involve the informal fallacy of shifting the context. In Section 4 we consider the possibility of applying the contextualist resolution of the general transitivity puzzle to Sorites arguments in particular, discussing the views of Kamp, Pinkal and Soames. Our negative conclusion, developed in Section 5, is that the paradoxes can be formulated in a way that does not commit the informal fallacy: context is held fixed. In the final section, we suggest a different defense against the objection used in Section 2.

\section{Introduction}

A Sorites paradox is an argument whose form is like this (the Sorites scheme):

$$
\begin{aligned}
& p_{\mathrm{o}} \\
& p_{\mathrm{o}} \rightarrow p_{1} \\
& \vdots \quad \vdots \\
& p_{n-1} \rightarrow \\
\therefore & p_{n}
\end{aligned}
$$

There is also a pure conditional variant of the Sorites scheme, in which the minor premise is discarded and the conclusion is ' $p_{0} \rightarrow p_{n}{ }^{\top}$ :

$$
\begin{gathered}
p_{0} \rightarrow p_{1} \\
\vdots \quad \vdots \\
\quad p_{n-1} \rightarrow p_{n} \\
\therefore \quad p_{0} \rightarrow p_{n}
\end{gathered}
$$


What makes certain instances of either Sorites scheme paradoxical is that in them, $p_{\mathrm{o}}$ is true and $p_{n}$ is false, while because of the nature of the vocabulary in each $p_{i}$, and empirical facts about the objects mentioned in each $p_{i}$, each conditional premise of the form ${ }^{\ulcorner} p_{m-1} \rightarrow p_{m}{ }^{\top}$ also seems true. Its seeming true is a result of its employing a tolerant concept, that is, a concept meeting the following condition: (i) the applicability of the concept to an object or in a situation depends on certain (perhaps only roughly) quantifiable features of the object or situation; (ii) significantly different quantities of these features can make an absolute difference between applicability and inapplicability of the concept; but (iii) there are differences in the quantities of these features of a magnitude too small to 'affect the justice with which [the concept] applies to a particular case' (Wright 1976:156-7). The problem is that insignificant differences can accumulate across cases into a significant difference.

Take, for example, the concept of being well-paid ${ }_{C}$ by one's employer, where $C$ is a reference-class and we are concerned only with individuals belonging to it; $C$ might be, say, the class of Full Professors in humanities departments in the current US News and World Report top 50 research universities in the USA. It seems plausible that if any member of this group is well-paid, then any other member of the group who is paid less, but no more than $\$ 100$ less, than the given member, is also well-paid $_{C}$ (make it $\$ 10$ or $\$ 1$ if you have doubts about $\$ 100$ ). Suppose we agree as well that members of $C$ are well-paid ${ }_{C}$ if they are paid $\$ 150,000$ or more, and not well-paid ${ }_{C}$ if they are paid $\$ 75,000$ or less. Then if $a_{0}, \ldots, a_{750}$ are 751 members of $C$ such that $a_{\mathrm{o}}$ is paid $\$ 150,000$ and $a_{i}$ is paid $\$ 100$ more than $a_{i+1}$, we have an instance of the Sorites scheme that leads to a contradiction. For by our principles, ' $a_{0}$ is wellpaid $_{C}$ ' is true, and each conditional ' $a_{i}$ is well-paid ${ }_{C} \rightarrow a_{i+1}$ is well-paid ${ }_{C}$ ' is true, so 
' $a_{750}$ is well-paid ${ }_{C}$ ' must be true as well; but $a_{750}$ makes $\$ 75,000$, which means $a_{750}$ is not well-paid ${ }_{C}$. And the pure conditional version yields the conclusion ' $a_{\mathrm{o}}$ is wellpaid $_{C} \rightarrow a_{750}$ is well-paid ${ }_{C}^{\prime}$, which is false. But chaining conditionals cannot lead from truths to falsehood.

There are only a few options for a resolution of this problem: (i) at least one conditional premise in the sequence is untrue, ${ }^{1}$ which, since there are only finitely many premises, means that some conditional premise is the first untrue premise; or (ii) there is something wrong with the logic; or (iii) there is some kind of semantic problem with the premises, such as an equivocation, which renders the logic inapplicable. In addition, any plausible resolution will have to include a good explanation of why the untrue conditionals seem true, or why the logic seems unassailable, or why the equivocation goes unnoticed. We take the options in turn. ${ }^{2}$

\section{Untrue Premises}

There are many attempts at solving the Sorites which claim to show that at least one conditional premise is untrue. The most conservative, epistemicism, says that there is a first premise in the list with a true antecedent and a false consequent, but it is impossible to know which premise it is, because it is impossible to know where the cut-off for being well-paid ${ }_{C}$ lies. ${ }^{3}$ The postulation of a sharp boundary at which a predicate like 'well-paid ${ }_{C}$ ' abruptly ceases to apply, a boundary (dollar value, in this case) that is in principle unknowable, is the feature of epistemicism

\footnotetext{
${ }^{1}$ I use 'untrue' as a catch-all, covering 'intermediate or false', 'neither true nor false, or false', 'not wholly true', 'not supertrue', and so on.

${ }^{2}$ Another constraint on a solution, which I will not directly address here, arises from an observation often made by Wright, namely, that the paradoxical arguments seem just as puzzling if the conditional premises are replaced by premises of the form $\neg(p \wedge \neg q)$; see, e.g., (Wright 1987, passim). (Edgington 1996) develops apparatus which addresses this; see further (Forbes 2010:429, n.11).

${ }^{3}$ For epistemicism, see especially (Sorensen 1988) and (Williamson 1994).
} 
that generates the greatest resistance to it. But I will not attempt to evaluate the debunking explanation epistemicists give of the natural view that there is no such sharp boundary, ${ }^{4}$ since the first thing I want to suggest here is that the problem for epistemicists is a problem for everyone who holds that some conditional premise in a Sorites is untrue.

It is hard to believe in the epistemicist's sharp boundaries because there appears to be nothing in virtue of which such a boundary could come to be. For there is no discontinuity in nature to mark the extension of a vague concept or predicate; nor is there some Board of Standards entitled to stipulate a boundary (except in cases like giving a legal definition of 'adult', which introduces a special sense of the word for special contexts). And there is no reason to think that the actual pattern of usage of a predicate like 'well-paid ${ }_{C}$ ' in a community will somehow determine a sharp boundary. ${ }^{5}$ Perhaps there is no reason at all why there is a sharp boundary, but there is one nevertheless. Perhaps, for example, a full professor in a humanities department in the current $U S N \& W R$ top 50 national research universities is well-paid ${ }_{C}$ iff he or she has a salary of at least $\$ 92,367.41$, and that's just the way it is. That this is not really intelligible is the main objection to epistemicism. ${ }^{6}$

In the previous paragraph we have given an argument against sharp boundaries, not just cited an intuition: there must be an explanation why there is a sharp boundary, an explanation of how such a boundary comes to exist, but none of the possible grounds for such a boundary obtains. However, this argument applies equally well against other views about how there comes to be a first untrue prem-

\footnotetext{
${ }^{4}$ See (Wright 2003:87) for some negative comment with which I am in agreement.

${ }^{5}$ Williamson (1994) argues that the meaning of a vague predicate supervenes on its use, which may be true. But it is a further question whether the meaning includes a sharp boundary and whether the pattern of usage fixes where that boundary is. There is no reason to think that it must, even if, by luck, it does.

${ }^{6}$ Two questions should be distinguished. One is why a sharp cut-off exists at all, another is why it falls where it does. I am arguing that there is no good explanation why a sharp cut-off exists. If there were, there might still be no explanation why it falls here rather than there. But that would be less objectionable.
} 
ise in a Sorites. Such a premise must have a true antecedent, and for a conditional with a true antecedent to be untrue, on any view, is for its consequent to have a different semantic status than its antecedent. ${ }^{7}$ So there will be an abrupt switch from the dollar values of salaries that put professors in the extension of 'well-paid ${ }_{C}$ ' to dollar values that do not. We may be evaluating the conditional 'If Professor X is well-paid ${ }_{C}$ then Professor Y is well-paid ${ }_{C}$ in which the antecedent is true, or wholly true, or determinately true, while the consequent is not. So the specific $\$ 100$ ( $\$ 10$, \$1) drop from the salary of Professor X to that of Professor Y marks a semantically significant transition. It's not a transition from true to false, certainly, but where it comes from is just as mysterious. For as before, there is no discontinuity in nature to mark the point of the transition; nor is there some Board of Standards entitled to stipulate the point at which 'well-paid ${ }_{C}$ ' becomes, say, neither true nor false of a wage earner in $C$. And there is no reason to think that the actual pattern of usage of a predicate like 'well-paid, in a community will somehow determine a precise point of transition. That there is such a point appears to be a fiction. ${ }^{8}$

\footnotetext{
${ }^{7}$ On supervaluationism, the consequent of the first non-supertrue premise is the first consequent for which there is an admissible sharpening making it false. See (Kamp 1981:253-4) for criticism of supervaluationism for introducing indefensible sharp distinctions.

${ }^{8}$ See (Sainsbury 1991:9-15; also in Keefe and P. Smith 1996:257-63) for eloquent exposition of this theme. However, N. Smith (2008:308-15) suggests that some precise points of transition can be determined by vote. In the present case, we simply survey language-users, and although they may disagree about what salaries make you well-paid ${ }_{C}$, we can be sure that there is a least salary which they unanimously agree makes you well-paid ${ }_{C}$. So we might say that only if you earn less than that salary does the claim that you are well-paid $_{C}$ fail to be unqualifiedly true. I have three doubts about this. First, while $100 \%$ is a nice round number, $90 \%$ is almost as nice: what makes it the case that unanimity is the correct requirement? Second, we need a criterion for excluding the judgements of certain voters, e.g., those who have had one drink too many (Robertson 2000:331) and make judgements that diverge wildly from those of the vast majority (that can't be the criterion for Smith's purposes, because it is vague - 'wildly', 'vast'). Third, the envisaged polls are counterfactual: we are talking about what language-users would say if surveyed. But then the least

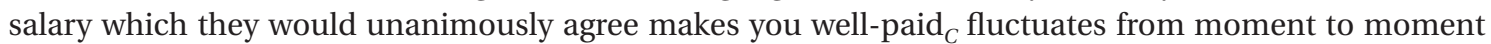
(not just, as Smith allows, from today to tomorrow), since judgements about the cut-off may be sensitive to arbitrary factors, e.g., the current atmospheric pressure or wind strength. This makes it hard to see how the vote is reflecting some fact about how little you can be paid and still be well-paid ${ }_{C}$.
} 


\section{Faulty Logic}

Perhaps a Sorites paradox can be taken as a refutation of the principles of classical logic on which the truth of its conclusion depends: there may be a problem either with modus ponens, or, for the pure conditional paradox, with transitivity of ' $\rightarrow$ '. Indeed, some semantics that render at least one premise untrue involve apparatus that can also be used to make trouble for these principles. For example, in fuzzy logic, the usual approach involves using the real interval $[0,1]$ for degrees of truth from wholly false to wholly true, along with an account of ' $\rightarrow$ ' on which the degree of truth of a conditional drops as the gap between the higher degree of truth of the antecedent and the lower degree of truth of the consequent widens, until we reach the limiting case when antecedent is $1(T)$ and consequent is $O(\perp)$, which results in the lowest possible degree of truth, $o$. The simplest clause with this effect is

(1) $\quad v[p \rightarrow q]=1-(\nu[p]-v[q]) .^{9}$

But (1) makes modus ponens and transitivity of ' $\rightarrow$ ' invalid on an account of validity that generalizes classical validity in the way that (1) generalizes material implication (whose table is the special case when $[0,1]$ is replaced by $\{0,1\}$ ). Suppose we define $\models_{g}$ for finite premise sets by:

(2) $\quad p_{1}, \ldots, p_{n} \vDash_{g} q$ iff $v[q] \geqslant \min \left\{v\left[p_{1}\right], \ldots, v\left[p_{n}\right]\right\}$.

In other words, a valid argument-form is one in which, for any interpretation $v$, the degree of truth of the conclusion on $v$ is no lower than that of the least true premise on $v$. But then, if $v(\mathrm{~A})=.9, v(\mathrm{~B})=.8$, and $v(\mathrm{C})=.7$, we have by (1) and (2) that $\mathrm{A}, \mathrm{A} \rightarrow \mathrm{B} \#_{g} \mathrm{~B}$ and $\mathrm{A} \rightarrow \mathrm{B}, \mathrm{B} \rightarrow \mathrm{C} \neq_{g} \mathrm{~A} \rightarrow \mathrm{C}$. So the original Sorites schema is invalid,

\footnotetext{
${ }^{9} \cdot-$ ' is cut-off subtraction, $a-b=a-b$ if $a \geqslant b$, = o if $a<b$.
} 
as is the equally paradoxical pure conditional variant.

However, classifying the Sorites scheme as invalid is not essential to the fuzzy logic solution of the paradox. Modus ponens and transitivity of ' $\rightarrow$ ' are restored by a more orthodox account of validity, where we say $p_{1}, \ldots, p_{n} \vDash q$ iff $q$ is wholly true on every interpretation on which $p_{1}, \ldots, p_{n}$ are all wholly true $\left(v\left[p_{i}\right]=1\right)$. But some of the apparently true conditional premises of a relevant instance of the scheme will still be slightly less than wholly true, according to this account. That they are slightly less than wholly true makes the argument unsound, and that they are slightly less than wholly true explains why we are inclined to take them to be true. So a complete solution of the paradox is available without challenging its logic. But, like other multivalued and supervaluationist solutions, this comes at the price of positing sharp boundaries, for example, the one marking the least salary $\$ n$ such that if $x$ earns $\$ n$ it is wholly true that $x$ is well-paid ${ }_{C}{ }^{10}$

A solution which only challenges the logic has the prima facie possibility of blocking the paradox without introducing sharp boundaries. But if we look to critiques of classical conditional logic which are not motivated by considerations about vagueness for the ingredients of an analogous critique of Sorites logic, we are liable to be disappointed.

Suppose that Seb and Steve are two athletes who are about equally as good as each other at a certain event, say men's 1500m track, and suppose also that they are far ahead of the rest of the competition. In this circumstance, the following conditional, concerning the Olympics in which they both compete at the peak of

\footnotetext{
${ }^{10}$ This price is also paid by the version of validity on which modus ponens and transitivity are invalid. And if we are generally explaining misjudgment (including misjudgments about validity) in terms of failure to notice very small differences, this version will seem unattractive on another ground, namely, that the conclusion of a modus ponens or transitivity inference can be much more false than its least true premise; e.g., two conditional premises for an application of transitivity that each have degree of truth .7 produce a conclusion with degree of truth .4. Surely we'd notice that? See further (Williamson 1994:124).
} 
their powers, seems true:

(3) If Steve wins the gold, Seb will win the silver.

Again, since they have no real rivals other than each other, it also seems true that

(4) If injury forces Seb to withdraw, Steve will win the gold.

But if we chain (4) and (3), the result is the surely false

(5) If injury forces Seb to withdraw, Seb will win the silver.

Of course, on the material reading, if (5) is false, i.e., if injury forces Seb to withdraw and he doesn't win the silver, then (3) and (4) are incompatible, and in view of (4), one might be tempted to revise the judgement that (3) is true. But if we make revisions for that sort of reason, the outcome will be that no conditional is true unless its antecedent strictly implies its consequent; for example, combine (4) with the evidently true 'if injury forces both Seb and Steve to withdraw, then injury forces Seb to withdraw'. So (4) is false too, and by the same token, most of the conditionals we ordinarily assert are false. But any philosophical analysis of some locution is highly suspect if it says that speakers who understand the locution and aim to speak the truth using it nevertheless typically produce falsehoods as a result of using it, no matter how expert they are about the subject-matter. It's much more likely that there is some error in the analysis.

A better account of what is going on in the above inference involves appeal to the context in which a conditional is evaluated. We may suppose that with each such context there is associated a set of admissible possible worlds, and the truthcondition for a conditional in a context $\mathrm{T}$ is that its consequent is true in every $\mathrm{T}$ - 
admissible world in which its antecedent is true. So we can say that in evaluating (3) as true we are in a context in whose admissible worlds both Seb and Steve run in the final (and where other background conditions are as close as possible to the actual world). But the antecedent of (4), for pragmatic reasons, puts us in a context with a wider class of admissible worlds, including some in which only Steve runs. Relative to this wider class, (3) is false, since if Steve gets the gold in a race without Seb, one of the less talented others will have picked up the silver. And in the narrower class of worlds, where both run, (5) has an impossible antecedent, which, at least arguably, suffices for its truth.

On this analysis, there is no real threat to transitivity in (3)-(5). For if the truthvalues of premises in an argument may vary with context, demonstrations of validity or invalidity require the context to be held fixed; while as we have just seen, we get (3) and (4) both true only when we let the context change from premise to premise. That the transitivity scheme turns out to be valid is an advantage for the hypothesis of context-dependency over other semantics which simply accept, in the light of cases like Seb-Steve, that it is invalid. For it is hard to see how it could be invalid, if a conditional asserts the sufficiency of the antecedent for the consequent.

How, exactly, are we to model the context-dependency we are attributing here? A very simple account takes the domain of worlds of the context as a domain for the interpretation of modal operators. The conditionals in (3)-(5) are then analyzed as strict conditionals, that is, as the necessitations of material conditionals, formulae of the form ${ }^{\top} \square_{\mathrm{T}}(p \supset q){ }^{\top}$, in which $\square_{\mathrm{T}}$ expresses universal quantification over the domain of worlds of the context $\mathrm{T}$. If context determines the relevant domain of discourse for $\square_{T}$, the switch in moving from (3) to (4) is like the switch that occurs when two professors report on how the honors students did in their classes. 
If Professor X reports 'every honors students got an A' and Professor Y reports 'not every honors student got an A', there is no contradiction, since the domain of the restricted quantifier 'every honors student' changes from report to report. In the same way, the domain of $\square_{T}$ changes from (3) to (4), and when (5) gets its intuitively correct evaluation as false, we are in a domain in which (3) is false.

\section{Context and Sorites Conditionals}

(3), (4) and (5) constitute a paradox only if we fail to notice switches in context. Granted that the example doesn't provide a reason to reject transitivity, might the grounds it provides for rejecting the argument as an instance of transitivity be generalizable to at least the pure conditional Sorites scheme, or both schemes? That is, can we make a similar analysis of puzzling instances of the schemes, to the effect that the premises are certainly all true, but each is true in a certain context, and the relevant context shifts at various points as we go through the premises? Approaches to the Sorites embodying this diagnosis are to be found in (Kamp 1981), (Pinkal 1984), and, the treatment which will be our main focus here, (Soames 1999). ${ }^{11}$

Soames's idea is that for a given vague predicate $F$, such as 'well-paid ${ }_{C}$, ${ }^{12}$ there is a default context $T_{0}$ (in Pinkal 1984, the 'basic' or 'root' interpretation, in Kamp

\footnotetext{
${ }^{11}$ We focus on Soames's approach because it is prima facie the most conservative of the three cited. Pinkal (who acknowledges Kamp 1981) is mainly concerned to develop a notion of 'practical consistency' which can be used to resist the Dummett-Wright charge of incoherence in language (Dummett 1975; Wright 1975), and this turns out to involve non-transitive entailment (Pinkal 1984:338). And Kamp's notion of (absolute, complete) truth in a context is such that it needn't be closed under modus ponens (see (a), (c) in Kamp 1981:260). If Soames' version of contextualism were successful, it would show that Kamp's and Pinkal's approaches involve more complexity and revisionism than is needed to solve the problem.

${ }^{12}$ Soames' running example of a vague predicate is 'looks green', but it is clear that he intends his analysis to apply to 'bald', 'green', 'well-paid', and so on, not just subjectivized versions like 'looks green' and 'seems bald'. However, I am in agreement with Edgington (1996:309, n.15) that the subjectivized predicates are a special case, for which an account in terms of context-dependency (perhaps in the style of Raffman 1994) may be appropriate in a way that it is not for the non-subjectivized predicates. Raffman's contextualism is rather different from the logical kinds under discussion here; see further (Forbes 2010:424, n.6).
} 
1981:253, 256 the 'minimal' context) in which the meaning of $F$ provides $F$ with a default extension, a default antiextension (Kamp uses 'positive extension' and 'negative extension'), and what Robertson (2000:332) calls an 'inextension'; in the case of 'well-paid ${ }_{C}$ ' the extension in $T_{0}$ contains the professors in $C$ which the rules of language combined with the empirical facts determine to be well-paid ${ }_{C}$; the antiextension contains the professors in $C$ which the rules of language plus the empirical facts determine to be non-well-paid ${ }_{C}$; and the inextension contains the professors in $C$ which the rules of language combined with the empirical facts are silent on they are the elements of $C$ for which 'well-paid ${ }_{C}$ ' is undefined.

However, in the course of a conversation, it is permissible for the participants to extend the extension (or antiextension) of $F$ in certain ways, for example by decreeing that such-and-such an object, hitherto in the inextension, is to be counted as $F$; for example, it might be stated that to be paid $\$ 90,000$ is to be well-paid ${ }_{C}$. Barring objections, 'well-paid ${ }_{C}$ ' will now have those members of $C$ earning at least \$90,00o in its extension, but it will also have in its extension those members of $C$ earning a sum less than $\$ 90,000$ but within the tolerance-range of ' well-paid $_{C}$ '. Earlier, we suggested that $\$ 100$ is within this range (at the likely cost of running out of actual professors, $\$ 10$ or $\$ 1$ could be used instead). So, by stipulating that to be paid $\$ 90,000$ is to be well-paid ${ }_{C}$, we have changed the extension of 'well-paid ${ }_{C}$ ' to include those in $C$ who earn at least $\$ 89,900$. Now, if tolerance for ' well-paid ${ }_{C}$ ' implies that anyone earning $\$ 100$ less than someone who is well-paid ${ }_{C}$ is also well-paid ${ }_{C}$, then we could not stop at $\$ 89,900$ : it would turn out, after sufficiently many steps, that someone who works pro bono is well-paid ${ }_{C}$. But the contextualist conception is rather different: what makes a predicate $F_{C}$ tolerant is that for any item $x \in C$ in the inextension of $F_{C}$, if $x$ is explicitly characterized in the context as being $F_{C}$ 
(Soames) or if its being $F_{C}$ is part of the background of the context (Kamp), or if $x$ is focused under the aspect of being $F_{C}$ (Pinkal), then the extension of $F_{C}$ expands to include everything that has at least the magnitude of $F_{C}$-making features that $x$ has, and also those items which do not have that magnitude, but whose shortfall is within the tolerance range (mutatis mutandis for 'non- $F_{C}$ ' and the antiextension of $F_{C}$ ). However, until one of these falling-insignificantly-short items $y$ is explicitly characterized as being $F_{C}$, or until the background is updated wth the judgement that $y$ is $F_{C}$, or until $y$ is focused under the aspect of being $F_{C}$, there is no way of iterating the extension-expanding principle by applying it over again to $y$. So we do not, in our example, end up concluding that those who work for nothing are in the extension of 'well-paid ${ }_{C}$ '.

The change in extension of $F$ consequent upon an accepted proposal that $x$ be taken to be $F$ (or an updating of the background with ' $F x$ ', etc.) is a change in the standards for being $F$, or more generally, a change in context. Now suppose someone reasons through our running instance of the Sorites scheme in the following way (the modus ponens walk-through): Professor $\mathrm{X}_{1}$ is well-paid ${ }_{C}$; if Professor $\mathrm{X}_{1}$ is well-paid ${ }_{C}$ then Professor $\mathrm{X}_{2}$ is well-paid w $_{C}$ so, Professor $\mathrm{X}_{2}$ is well-paid ${ }_{C}$. But if Professor $\mathrm{X}_{2}$ is well-paid ${ }_{C}$ then Professor $\mathrm{X}_{3}$ is well-paid ${ }_{C}$, and Professor $\mathrm{X}_{2}$ is wellpaid $_{C}$; so, Professor $\mathrm{X}_{3}$ is well-paid ${ }_{C}$; so...so Professor $\mathrm{X}_{n}$ (who works pro bono) is well-paid ${ }_{C}$. Each conditional $p \rightarrow q$ is true because, (i), according to the standards for being well-paid ${ }_{C}$ in force in the context $T$ in which the conditional is asserted, $p$ is true, either by virtue of being default-true (true in $T_{0}$ ), or by virtue of an expansion of the extension of 'well-paid ${ }_{C}$ ' which occurred when at the previous step $p$ was detached by modus ponens and asserted (the detachment and the assertion may be distinguished, if desired); and (ii) $q$ is true, because each professor is paid 
at most $\$ 100(\$ 10, \$ 1)$ more than the next one, so the professor mentioned in $q$ falls

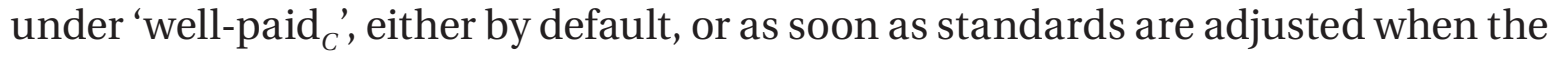
professor mentioned in $p$ is asserted, at the conclusion of the previous step, to be

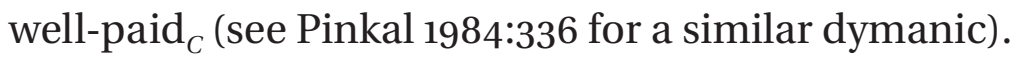

We therefore have a close parallel with the case of Seb and Steve: each conditional, in its own context, is true, but the argument, which seems to be a correct formal proof, is in fact fallacious because context sometimes changes from one step to another. In a genuinely correct formal proof, context is held fixed, or else the proof is carried out in a special formalism with a method of tracking change of context. In the present case, we think we have a genuine formal proof, because we fail to notice, or understand, how beyond a certain point each step in the walkthrough effects a context-change, a change in standards for the application of the relevant vague predicate.

There are, I think, some difficulties for this account. First, the diagnosis which Soames offers of the appearance of truth in each Sorites conditional is unpersuasive. According to the degree theorist, each conditional seems true either because it is true, or because it is so close to being true that it's entirely understandable that it is taken as true. So an understandable mistake is attributed. However, on Soames' view (Soames 1999:215), the mistake is not so understandable: for a vague empirical predicate $F$ we are said to confuse a principle such as

(6) If $x$ is $F$ and $y$ differs from $x$ in respect of $F$-ness only to an empirically indiscriminable degree, then $y$ is $F$

with a metalinguistic principle along the lines of 
(7) Anyone who characterizes $x$ as $F$ is committed to a standard that counts $y$ as being $F$ too, when $y$ differs from $x$ in respect of $F$-ness only to an empirically indiscriminable degree.

(6) concerns when objects must agree on whether or not they are $F$, while (7) concerns the commitments of speakers consequent upon making certain judgements, so on the face of it they are rather dissimilar. But perhaps we wouldn't realize that it is (7) that is driving Sorites reasoning until it is pointed out to us. However, there is little reason to think that the judgements of sophisticated speakers about Sorites conditionals are really confused versions of (7), in which they assent to if $x$ is $F$ then $y$ is $F$ ' when what they are really thinking is 'if I say $x$ is $F$ then I'm committed to a standard under which $y$ is $F$, or that they fail to notice that their reason for thinking the former is only a reason for thinking the latter. Surely, if $x$ is green and there is no perceptible difference in color between $x$ and $y$, then $y$ is green too; if $x \in C$ is wellpaid $_{C}$, and though $y \in C$ is paid a little less the difference wouldn't buy you anything at the local Five \& Dime, then $y$ is also well-paid ${ }_{C}$. These are highly plausible claims in their own right, and produce hesitation only in those who foresee a Sorites paradox coming down the tracks at them. ${ }^{13}$

A second, more severe objection, from (Robertson 2000), is that Soames' theory suffers from a spillover problem. If Professor Y is in the inextension of 'well-paid ${ }_{C}$ ', then it's a legitimate move to stipulate that Professor $Y$ is well-paid ${ }_{C}$. But it's conceivable that Professor Y is paid only a few dollars more than the highest-paid professor in the default antiextension of 'well-paid ${ }_{C}$ ', a certain Professor Z. And when we stipulate that Professor Y is well-paid ${ }_{C}$, we add to the extension of 'well-paid ${ }_{C}$ '

\footnotetext{
${ }^{13}$ I would make a similar response to Pinkal's suggestion (1984:330) that observational indistinguishability of $x$ and $y$ only guarantees truth-functional equivalence of observational predications of $x$ and $y$ when one or other of $x$ or $y$ is focused under the aspect of the observational predicate in question. There is no reason to think that in our confusion, the qualification about focus is simply something we overlook the need for.
} 
not only those who earn exactly what Professor Y does, but also those who earn a few dollars less. So Professor Z gets added. But no member of the default antiexten-

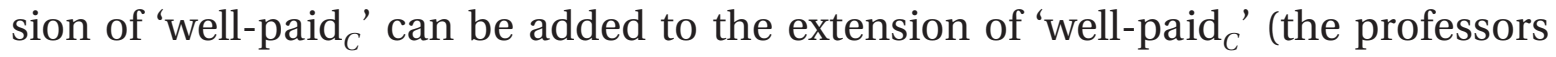
in the default antiextension are, if you like, the definitely not well-paid ${ }_{C}$ ones). So Soames' apparatus generates a contradiction. ${ }^{14}$

Perhaps this problem arises because Soames takes there to be sharp boundaries that delineate three groups, default extension, default inextension, and default antiextension. But this doesn't seem to be the crux of the matter. For it is of no help to change three to five by adding default 'buffer' zones between extension and inextension and inextension and antiextension. Presumably buffer-zone professors can be stipulated to be well-paid ${ }_{C}$ or not well-paid ${ }_{C}$, and so can be absorbed into the extension or antiextension of 'well-paid ${ }_{C}$ '. Thus we quickly find ourselves back at the spillover point. It also does not help to blur the sharp boundaries that delineate default extension, inextension and antiextension, say by taking the rules of language that govern 'well-paid ${ }_{C}$ ' themselves to involve vague terminology. We might agree that to be well-paid ${ }_{C}$ it's enough to be paid an amount that is close to the salary of some person who is well-paid ${ }_{C}$, or about the same as the salary of such a person. But so long as, for each salary, there are lesser salaries that are close to or about the same as the given one, and about the same amount less across cases, we can simply advance in the style of Robertson (op. cit., 332-3) to a professor, $x$, who is, in the current context, well-paid ${ }_{C}$, such that there is a lesser-paid professor who is default not well-paid ${ }_{C}$, but whose salary is close to or about the same as that of $x$.

The spillover problem therefore appears to be a serious one. Maybe it can be

\footnotetext{
${ }^{14}$ Analogous problems appear to afflict the other contextualisms. For example, Kamp (1981:260) has a notion of coherence on which some contexts are coherent, some incoherent, and some neither. When we announce that Professor Y is well-paid, we are in an incoherent context, but it's unclear what's to stop us reasoning our way into it from coherent contexts by a modus ponens walkthrough.
} 
met by suitable principles, though it seems likely that the motivation for such principles will only be that they avoid the problem. ${ }^{15}$ However, I wish here to pursue another difficulty, which I think vitiates any kind of contextualist approach to the Sorites, that is, any approach that explains the force of a Sorites in terms of the truth of each conditional in its own context, with shifts in context rendering the arguments fallacious and the subtle nature of the shifts explaining away the impression that the premises are all true together. For if it is possible to fix the context in a way that retains the appearance that the premises are all true together, their appearing that way isn't explained away by shifts in context; and without shifts in context, the contextualist has no grounds to say that the arguments are fallacious.

\footnotetext{
${ }^{15}$ In his response to Robertson, Soames (2002:443-4, n.13) agrees that his principles will generate Robertson's contradiction from a case of objects $y$ and $z$ such that $y$ is in the default inextension of 'looks green' and $z$ is in the default antiextension, $y$ and $z$ are perceptually indistinguishable as regards color, and in the context $c$ the speaker $s$ asserts 'that looks green', demonstrating $y$. To repair the problem, Soames suggests that 'the two most promising alternatives' are as follows. (A1): $s$ 's assertion 'that looks green' in $c$ does not semantically express any proposition in $c$ because 'looks green' does not semantically express any property in $c$, but $s$ does succeed in asserting a proposition, namely, one which attributes to $y$ a property we can call looking green*, which applies to anything perceptually indistinguishable from $y$ as regards color. By this characterization, $z$ looks green*. But that is unfortunate, since our intuition about the case is not merely that $s$ seems to assert something in $c$, but that, being a normal speaker, what $s$ asserts of $y$ in $c$ with 'looks green' is not true of $z$; for after all, $z$ doesn't look green, it only looks green*. In other words, it's counterintuitive that one can use 'looks green' in $c$ to correctly attribute a property possessed by something that doesn't look green by any acceptable standards. This makes (A1) rather unappealing. (A2): $s$ 's assertion 'that looks green' semantically expresses a proposition in $c$, but the property expressed by 'looks green' in $c$ isn't the result of adjustment consequent upon the assertion. Rather, it's the property $P_{x}$ expressed by 'looks green' in 'that looks green' in the context where 'that' denotes the object $x$ immediately before $y$ in the Sorites series ( $P_{x}$ has in its extension $x$ and everything that looks no less green than $x$, including $y$ ). But this does not resolve the contradiction. If stipulating in $c$ that $x$ looks green is to put $y$ but not $z$ into the $c$-extension of 'looks green', $x$ and $z$ must be discriminable, while neither $x$ and $y$ nor $y$ and $z$ are. Therefore, there should be a perfectly coherent context $c^{*}$ in which $x$ is stipulated to look green while $z$ (which is default not-green-looking) is announced not to look green. By the stipulation, $y$ looks green (it is indiscriminable from $x$ ), and by the announcement, $y$ doesn't look green (it is indiscriminable from $z$ ), both in the same context. So if $c^{*}$ is not just to be ruled out of order by decree, it seems option A2 should be revised to say that 'looks green' in 'that looks green', 'that' denoting $y$, expresses $P_{w}$ in $c$, where $w$ is immediately before $x$ in the Sorites series and is indistinguishable from $x$ and distinguishable from $y$. So (i) the demonstrative utterance may well be untrue, even though, one wants to say, $y$ really does look green to $s$. And (ii) there are no acceptable standards under which there is a property of looking green that $y$ has. Consequently, if we say 'that looks green' denoting $x$, we can't be semantically expressing $P_{x}$, but must instead be expressing $P_{w}$. It is not clear where this will end. (For discussion of what might be a related issue, see Soames 1999:222-3, n.11.)
} 


\section{Conditionals and the Sufficiency Relation}

For the purposes of this discussion, I focus on the pure conditional Sorites scheme. Each conditional in an instance of such a scheme is in fact derived from two other premises. For instance, the conditional 'if Professor $\mathrm{X}_{1}$ is well-paid ${ }_{C}$ then Professor

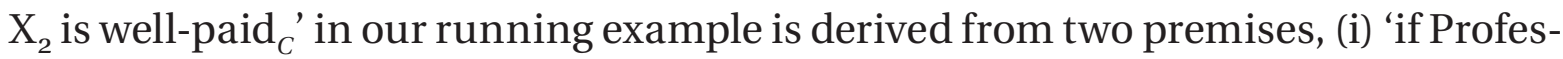
sor $\mathrm{X}_{1}$ is paid at most $\$ 100$ more than Professor $\mathrm{X}_{2}$, then if Professor $\mathrm{X}_{1}$ is well-paid ${ }_{C}$, Professor $\mathrm{X}_{2}$ is well-paid ${ }_{C}$ ', and (ii) 'Professor $\mathrm{X}_{1}$ is paid at most $\$ 100$ more than Professor $\mathrm{X}_{2}$. In other words, the premises of the pure conditional scheme are derived by an inference of the form $p \rightarrow(q \rightarrow r), p \vdash q \rightarrow r$, where $p$ is the relational premise that states that the next item differs only by such-and-such an amount, where the amount in question is within the tolerance range of the relevant predicate.

Orthodox accounts of the conditional are typically formulated in a metalanguage with material implication $(\supset)$ and quantification over indices of some sort (e.g., the non-variably strict s5 conditional $p \rightarrow q$ is defined as 'for all $w, p$ is true at $w \supset q$ is true at $\left.w^{\prime}\right)$. These analyses are not indicative of realistic strategies for establishing conditionals. It is more realistic to suppose that when we consider the major premise of the argument for a specific Sorites conditional, for example,

(8) If Professor $\mathrm{X}$ is paid at most $\$ 100$ more than Professor $\mathrm{Y}$, then if Profes-

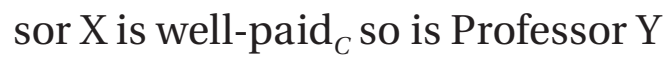

we apprehend a relation between antecedent ('Professor $\mathrm{X}$ is paid at most $\$ 100$ more than Professor Y') and (conditional) consequent that makes the main conditional in (8) true. The relation is that of sufficiency: the antecedent suffices for the consequent. The truth-condition of $p \rightarrow q$ is just that this relationship should hold between the propositions $p$ and $q$, something which can be established by a deri- 
vation of $q$ from $p$, but whose holding does not consist in such derivability.

There are competing accounts of sufficing, for instance, a material account, a strict account, and a relevant account. But there is a problem for contextualism independent of this choice. (8) and the other conditionals which, along with the relational premises, entail the premises of a Sorites, all seem equally good candidates for truth. In our current terms, this is to say that in each case it looks as if the relational premise is sufficient for the antecedent of the Sorites premise to suffice for its consequent: we perceive an a posteriori relation of sufficiency between propositions such as 'Professor $\mathrm{X}$ is paid at most $\$ 100$ more than Professor Y' and the proposition 'Professor Y is well-paid ${ }_{C}$ if Professor $X$ is well-paid $_{C}$ '. So in the latter conditional, the antecedent also suffices for the consequent. What is important here is that apprehension of sufficiency of its antecedent ('Professor $\mathrm{X}$ is wellpaid $_{C}$ ') for its consequent does not require any attitude of endorsement towards the antecedent. But this in turn means there is nothing in the apprehension of the truth of the premises (if they are all true) to trigger a change of context. So we have a fixed context in which all the premises of a Sorites seem equally true, because the relation of sufficiency seems to hold between antecedent and consequent in each conditional (given the relational premise). Hence it is not the case that they only all seem true because in evaluating each we implicitly shift to a context in which the conditional in question is true. And insofar as we are apprehending a sufficiency relationship between antecedent and consequent of the actual premises, we are not stumbling into some conflation of these premises with metalinguistic principles. Once again, then, the only recourse the contextualist has to block a Sorites is to insist that some premises are untrue, because of the mysterious sharp divisions between extension, inextension and antiextension of the vague predi- 
cate in question. ${ }^{16}$

There are other accounts of conditionals on which evaluating a conditional does involve taking an endorsing attitude towards the antecedent, perhaps thereby changing context. One such account is the 'suppositional' one developed in (Barnett 2006). On this account, 'if $p$ then $q$ ' is said to be synonymous with 'supposing that $p$, then $q$, and for a conditional to be true is for its consequent to be true under the supposition of its antecedent; that is, a conditional is true iff, supposing its antecedent to be true, its consequent is true as well. ${ }^{17}$ But even though this looks like it might lead to the conclusion that each premise is true in its own context, that is not so. For though supposing the antecedent changes the standards under which the consequent is evaluated, the supposition is cancelled when the truth-value of the consequent is transferred to the whole conditional; hence the resulting truthvalue is the truth-value in the default context. This means the semantics of the conditionals guarantees that the context is held fixed. For example, if 'Professor $\mathrm{X}_{121}$ is well-paid ${ }_{C}^{\prime}$ is default undefined, then

(9) If Professor $X_{121}$ is well-paid ${ }_{C}$ then Professor $X_{122}$ is well-paid ${ }_{C}$

is true in the default context, because when we evaluate 'Professor $\mathrm{X}_{122}$ is well-paid ${ }_{C}$ ' under the supposition that Professor $\mathrm{X}_{121}$ is well-paid ${ }_{C}$, we have changed the standards for being well-paid ${ }_{C}$ to ones under which Professor $\mathrm{X}_{121}$ is in the extension of

${ }^{16}$ Soames doesn't himself propose that context-shift explains why a standard Sorites (as opposed to a 'forced march') seems sound but is fallacious. He simply insists that one conditional must be untrue, and as described in $\$ 4$, posits an error thesis according to which we deny this because we confuse 'if $X$ is well-paid so is Y' with "if $\mathrm{X}$ is \{stipulated/assumed/agreed\} to be in the extension of 'well-paid' then Y is in it too". Pinkal's position (1984:338) is similar: we only advance towards the spillover point by mixing steps involving classical consequence with steps involving practical consequence, for if we restrict ourselves to classical consequence, the first conditional with a true antecedent and undefined consequent stops the reasoning.

${ }^{17}$ Barnett derives a non-classical logic for 'if...then' from this starting-point, but that doesn't appear to be intrinsic to the basic approach. He employs a very substantive notion of supposition, on which no moves can be made if a contradiction is supposed. On a more minimal notion of supposition, ex falso quodlibet could still be justified. 
'is well-paid ${ }_{C}^{\prime}$. This change in standards doesn't affect the relational premise, so

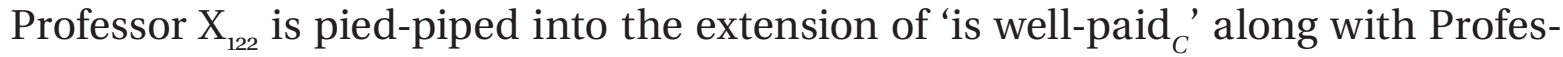
sor $\mathrm{X}_{121}$. Thus the consequent of (9) is true under the supposition of its antecedent, i.e., in the context created by supposing its antecedent. But this means that (9) as a whole is true in the default context, for its truth-condition in the default context is just that if we hypothesize a context that verifies the antecedent, the consequent also holds in that context. The same is true for all the other premises, so they are all true in the original context (the intuitively correct result). Hence, assuming transitivity, the absurd conclusion that if Professor $\mathrm{X}_{1}$ is well-paid ${ }_{C}$ then so is Professor $\mathrm{X}_{\text {pro bono, }}$ is also true in the default context.

On Soames' view, we should not accept that every premise is true in the original context. This is not because two are untrue, one marking the crossing of the lines between default extension and default inextension, and the other the line between default inextension and default antiextension. For in each case, the effect of supposing the antecedent is to move the extension/inextension boundary beyond the point where it could cause trouble, if it is not already beyond that point. The spoiler is again the spillover case: if Professor $\mathrm{X}_{m}$ is the least well-paid member of the default inextension, then the premise 'if Professor $X_{m}$ is well-paid ${ }_{C}$ then Professor $\mathrm{X}_{m+1}$ is well-paid ${ }_{C}^{\prime}$ won't be true even on the suppositional account, provided we have a principled reason why the pied-piping effect should fail for Professor $\mathrm{X}_{m+1}$.

But this is not a satisfactory way of responding to the problem. For it relies on there being a principled reason why the pied-piping effect should fail for Professor $\mathrm{X}_{m+1}$ (see note 15 for my scepticism that such a reason exists). Secondly, even if such a reason were forthcoming, we would still have a valid argument whose premises 
are true in the default context but whose conclusion, by Soames' lights, is untrue. For although we won't be able to conclude from the full instance of the pure conditional scheme that if Professor $\mathrm{X}_{1}$ is well-paid ${ }_{C}$ then so is Professor $\mathrm{X}_{\text {pro bono, }}$, we will be able to conclude from a truncated instance that if Professor $X_{1}$ is well-paid ${ }_{C}$ then so is Professor $\mathrm{X}_{m}$ (the least well-paid member of the default inextension).

The remaining move to make contextualism compatible with the suppositional account of conditionals is to formulate a semantics for conditionals on which transitivity fails. ${ }^{18}$ But the following reasoning suggests that transitivity should hold, at least in a range of cases our current ones fall into. For suppose that $q$ is the case on the supposition that $p$, and that $r$ is the case on the supposition that $q$. Then we may suppose that $p$, allowing us to conclude that $q$, and next, take whatever reasoning showed that $r$ is the case on the supposition that $q$, and use that reasoning to show that $r$ is the case on the supposition that $p$, applying it to the $q$ we inferred from the supposition that $p$.

The general form of argument here is unreliable, for it may be that while $r$ can be concluded when $q$ is supposed, it cannot be concluded when $q$ is inferred. A familiar example of this phenomenon is the sequent $A \vdash_{s 5} \square \diamond A$, where it seems that we should be able to assume A, infer $\diamond$ A by $\diamond$ I, then $\square \diamond A$ by $\square$ I. But $\square$ I requires that the formula it is applied to depends only on assumptions that are fully modalized (for a sentential language, $p$ is fully modalized iff every sentence letter in $p$ is within the scope of a $\square$ or $\diamond)$. Since A is not fully modalized, and $\diamond A$ depends on A, $\square$ I can't be used on $\diamond$ A. One solution is to combine two separate lines of reasoning: in the first we show that $\mathrm{A} \vdash \diamond \mathrm{A}$ by a use of $\diamond \mathrm{I}$, and in the second we show $\vdash \diamond \mathrm{A} \rightarrow \square \diamond \mathrm{A}$ by $\square \mathrm{I}$ and $\rightarrow \mathrm{I}$ (since $\diamond \mathrm{A}$ depends on itself and is fully modalized, $\square \mathrm{I}$

${ }^{18}$ (Barnett 2006) endorses a probabilistic semantics on which transitivity does fail (549-59), but I don't think this is well-motivated: the 'counterexamples' to transitivity are like the Seb-Steve case, for which there is an independently plausible diagnosis that preserves transitivity. 
can be applied to it). We get $\mathrm{A} \vdash \square \diamond \mathrm{A}$ by a final use of modus ponens.

Perhaps there is a comparable difficulty for our justification of transitivity:

(10) If Professor X is well-paid ${ }_{C}$ then Professor Y is well-paid ${ }_{C}$

says that Professor $\mathrm{Y}$ is well-paid ${ }_{C}$ supposing a context in which Professor $\mathrm{X}$ is wellpaid $_{C}$, while

(11) If Professor $Y$ is well-paid ${ }_{C}$ then Professor $Z$ is well-paid ${ }_{C}$

says that Professor $\mathrm{Z}$ is well-paid ${ }_{C}$ supposing a context in which Professor $Y$ is wellpaid $_{C}$. If we try to apply the reasoning that establishes (11) within the scope of a supposition of the antecedent of (10), we have moved the reasoning from one context into another, and this may be thought to be dubious. But as with the modal case, there is a way round the problem. We have reasoning which establishes (10) and reasoning which establishes (11), so we may make the supposition that $\mathrm{X}$ is well-paid $_{C}$ and within its scope suppose that $\mathrm{Y}$ is well-paid ${ }_{C}$. The reasoning that establishes (11) can now be applied, since the supposition-created context we are in is no different from the one created by the antecedent of (11). And once it's been established that $\mathrm{Z}$ is well-paid ${ }_{C}$ we can use $\rightarrow I$ to get (11) still within the scope of the supposition of the antecedent of (10). We may then apply modus ponens (we already have that $\mathrm{Y}$ is well-paid ${ }_{C}$ ) to conclude that $\mathrm{Z}$ is well-paid ${ }_{C}$, and a final $\rightarrow \mathrm{I}$ gets us

(12) If Professor $X$ is well-paid ${ }_{C}$ then Professor $Z$ is well-paid ${ }_{C}$

in the default context. So it looks as if transitivity is correct for this account of conditionals, meaning that if all the premises of a pure conditional Sorites scheme are true in the default context, so is the absurd conclusion. And the premises all seem 
equally true by the contextualist's own lights, given the suppositional analysis of what it takes to make them true. So it turns out that not even the suppositional account of conditionals eliminates the possibility of a single context in which all Sorites conditionals seem true. All the contextualist can say about this case is that the proofs are unsound, because there are untrue conditionals among their premises. The sharp boundary we hoped to avoid still confronts us.

\section{Conclusion}

So where does this leave us? The contextualist account is a version of the third option we distinguished at the end of $\$ 1$, according to which Sorites reasoning involves a fallacy of equivocation. For if it were true that there is no single context in which all the premises hold, we could think of the supposed changes in context (in standards) needed to evaluate all the premises as true, as changes in meaning. But if this third option leads to a dead end, we are thrown back on one of the other two.

I suggest that we ought to reconsider our argument in \$2 that any approach which classifies some premises as untrue is in the same boat as epistemicism, positing a sharp division whose existence is not intelligible. David Kaplan has drawn attention to a distinction between 'those features of a model which represent features of that which we model' and 'those features which are intrinsic to the model and play no representational role...artifacts of the model' (Kaplan 1975:722). ${ }^{19}$ For example, a scale model of HMS Victory, like Nelson's actual flagship, must have a physical length, but its value is an artifact of the model. It must also have a ratio of hull length to mainmast height, and this feature is representational, for the fur-

\footnotetext{
${ }^{19}$ In (Forbes 1984) I appealed to Kaplan's distinction to argue for the superiority of counterpart-theoretic over relativized possibility solutions to certain modal versions of Sorites paradoxes. I now think that this misapplies the distinction.
} 
ther it diverges from the ratio of the actual flagship's length to its mainmast height (roughly 7:5) the less accurate the model. ${ }^{20}$

In the same way, in a type-(i) formal model of a Sorites paradox (one that classifies it as valid but unsound) some specific premise must be the first untrue one. The question at issue is whether that premise's being the first untrue one is an artifact of the model, or rather, one of its representational features. For an epistemicist, its being the first untrue premise is a representational feature (identifying the wrong premise is like the model-builder who gets hull length to mainmast height wrong). The challenge for others, in particular, the degree theorist, is to make a case that, for each model and for whichever premise is the first untrue one on that model, it is only an artifact of the model that the premise in question is the first untrue one. Simultaneously, however, it has to be a representational feature of the model that some premise is the first untrue one. For on non-classical analyses of Sorites reasoning, though there is no fact of the matter which premise is the first untrue one, it is a fact that some premise is, since the conclusion is definitely false. Further investigation of the artifact/representation distinction may allow us to identify different types of sharp boundary, with epistemicism in sole possession of the least desirable. $^{21}$

Graeme Forbes Department of Philosophy University of Colorado Boulder, CO 80309

USA graeme.forbes@colorado.edu

\footnotetext{
${ }^{20}$ A model ship illustration is suggested in (Cook 2002:235). Cook's paper uses Kaplan's distinction to respond to some of the complaints about degree theory voiced in (Sainsbury 1991).

${ }^{21}$ I thank David Barnett, Ewan Klein, Teresa Robertson, Ben Rohrs and two anonymous referees for discussion and comments which helped improve this paper.
} 


\section{REFERENCES}

Barnett, D.: Zif is If. Mind 115, 519-565 (2006)

Beall, J. C., (ed.) Liars and Heaps. Oxford University Press (2003)

Cook, R.: Vagueness and Mathematical Precision. Mind 111, 225-247 (2002)

Dummett, M.: Wang's Paradox. Synthese 30, 301-324 (1975)

Edgington, D.: Vagueness by Degrees. In: Keefe and Smith (eds.), pp. 294-316. The MIT Press (1996)

Forbes, G.: Two Solutions to Chisholm’s Paradox. Philosophical Studies 41, 171-187 (1984)

Forbes, G.: Identity and the Facts of the Matter. In: Dietz, R., Moruzzi, S. (eds.) Cuts and Clouds: Vagueness, Its Nature and Its Logic, Oxford University Press (2010)

Kamp, H.: The Paradox of the Heap. In: Mönnich, U. (ed.) Aspects of Philosophical Logic, pp. 225277. D. Reidel (1981)

Kaplan, D.: How to Russell a Frege-Church. The Journal of Philosophy 72, 716-729 (1975)

Keefe, R., and Smith, P. (eds.) Vagueness: A Reader. The MIT Press (1996)

Pinkal, M.: Consistency and Context Change: The Sorites Paradox. In: Landman, F., Veltman, F. (eds.) Varieties of Formal Semantics, pp. 325-341. Foris (1984)

Raffman, D.: Vagueness Without Paradox. The Philosophical Review 103, 41-74 (1994)

Robertson, T.: On Soames's Solution to the Sorites Paradox. Analysis 60, 328-334 (2000)

Sainsbury, M.: Concepts Without Boundaries. Inaugural Lecture (1991), Stebbing Chair of Philosophy, King's College, London. Reprinted in Keefe and Smith 1996, pp. 251-264.

Smith, N. J. J.: Vagueness and Degrees of Truth. Oxford University Press (2008)

Soames, S.: Understanding Truth. Oxford University Press (1999)

Soames, S.: Replies. Philosophy and Phenomenological Research 65, 429-452 (2002)

Sorenson, R.: Blindspots. Oxford University Press (1988)

Williamson, T.: Vagueness. Routledge (1994) 
Wright, C.: Language-Mastery and the Sorites Paradox. In: Evans, G., McDowell, J. (eds.) Truth and Meaning, Oxford University Press (1976). Reprinted in Keefe and Smith 1996, pp. 151-173 (page references to the 1996 reprinting)

Wright, C.: Further Reflections on the Sorites Paradox. Philosophical Topics 15, 227-290 (1987)

Wright, C.: Vagueness: A Fifth Column Approach. In Beall 2003, pp. 84-105 (2003) 